\title{
Family history of diabetes in middle-aged Swedish men is a gender unrelated factor which associates with insulinopenia in newly diagnosed diabetic subjects
}

\author{
V. Grill, G. Persson, S. Carlsson, A. Norman, M. Alvarsson, C.-G. Östensson, L. Svanström, S. Efendic \\ Department of Molecular Medicine, Institute of Environmental Medicine and Department of Public Health Sciences, \\ Division of Social Medicine, Karolinska Institute, Stockholm, Sweden
}

\begin{abstract}
Summary We have investigated the association of a family history of diabetes with glucose tolerance in a population of Swedish men. All men 35-54 years of age in 1992 and living in four different local municipalities of the outer Stockholm area were screened by questionnaire. From 10236 completed questionnaires 1622 men, selected for presence of such a history but without known diabetes, as well as 1507 men without a family history underwent an oral glucose tolerance test. Diabetes (2 h-plasma glucose levels $>11.0 \mathrm{mmol} / \mathrm{l}$ ) was detected in 55 and impaired glucose tolerance (plasma glucose levels $7.8-11.0 \mathrm{mmol} / \mathrm{l})$ in 172 subjects. The odds ratio of diabetes, associated with a family history, was 4.1, confidence interval 2.1-8.3 and for impaired glucose tolerance 1.6, confidence interval 1.2-2.3. Influence of a family history was measurable also within the range of normal 2-h glucose concentrations: compared to 2 -h glucose levels $<3.8 \mathrm{mmol} / \mathrm{l}$; the odds ratio associated with a family history was 1.4 , confidence interval 1.1-1.7 and 1.3, confidence interval 1.1-1.6 for concentrations $4.8-5.7 \mathrm{mmol} / \mathrm{l}$ and $5.8-7.7 \mathrm{mmol} / \mathrm{l}$ respectively. The odds ratio of diabetes and impaired glucose tolerance among men with a family history increased with number and closeness of relatives with diabetes but was not affected by the gender of
\end{abstract}

the family member. Overweight $\left(\mathrm{BMI}>25.0 \mathrm{~kg} / \mathrm{m}^{2}\right)$ increased the odds ratio of diabetes in subjects with a family history, the odds ratio being 24 , confidence interval 3-177, when both conditions were present. In subjects with Type II (non-insulin-dependent) diabetes mellitus discovered during the investigation, the presence of a family history of diabetes was associated with decreased insulin secretion rather than insulin resistance as assessed by fasting insulin, homeostasis model assessment, and the 2-h insulin response to the oral glucose tolerance test. We conclude that a family history of diabetes strongly but independently of gender associates with decreased glucose tolerance. Furthermore, the results are compatible with a major role for low insulin secretion in the diabetogenic influence of a family history of diabetes in middle-aged Swedish men. Lastly, the very high risk for diabetes in middle-aged men with both a family history of diabetes and obesity indicates that such people should, for the purpose of therapeutic intervention, be identified in the general population. [Diabetologia (1999) 42:15-23]

Keywords Glucose tolerance, insulin secretion, insulin resistance, obesity, genetics of diabetes.
Received: 9 March 1998 and in revised form: 1 July 1998

Corresponding author: Dr. V. Grill, Department of Molecular Medicine, Karolinska Hospital, S-171 76 Stockholm, Sweden Abbreviations: OR, prevalence odds ratio; CI, confidence interval; IGT, impaired glucose tolerance; NGT, normal glucose tolerance; HOMA, homeostasis model assessment; OGTT, oral glucose tolerance test; WHR, waist/hip ratio.
Heredity is known to play an important part in the aetiology of Type II (non-insulin-dependent) diabetes mellitus [1]. The nature of the hereditary is, however, largely unclarified except in selected families in which, in some cases, single gene mutations have been discovered which can cause diabetes.

The role of heredity in the majority of subjects with Type II diabetes has been difficult to study due, in part, to the probable genotypic and phenotypic 
heterogeneity within and between different populations. Evolution of Type II diabetes has been extensively studied in populations with a high propensity for the disease, such as Pima Indians [2], inhabitants of South Pacific islands [3], or Mexican Americans [4]. The genetic background of diabetes in these populations, however, is bound to differ from that of less diabetes-prone ones which thus require separate investigation.

Studies of the impact of heredity in Type II diabetes in low-prevalence populations have been hampered by the small number of subjects with a family history of diabetes in the general population. The prevalence of diabetes in Sweden is low with 2-4\% of the population having diagnosed diabetes [5]. To circumvent the problem of the low prevalence of a family history of diabetes, we have carried out an epidemiological study in which a sample from the general population was enriched by subjects with a family history. The design of this study enabled us to assemble a sufficient number of subjects for detailed analysis of the impact of various types of family history and their relation to insulin secretion, insulin resistance and life-style risk factors, such as obesity.

In particular, the study population made it possible to analyse in detail a possible relation between a family history on maternal compared with paternal side for glucose tolerance and other diabetes-related variables in the probands. Such analysis is of special interest due to the divergent results reported. Many investigations thus show a preferential maternal effect [6-9] but others fail to do so [10].

The main questions we tested in our study were: 1 ) is there a strong association between a family history of diabetes and decreased glucose tolerance in a population representative of middle-aged Swedish men?,2) are there gender differences as to the impact of a family history on glucose tolerance, insulin sensitivity or insulin secretion or any of them?,3) to which extent does obesity interact with family history?,4) is family history as a diabetogenic factor associated with low insulin sensitivity or low insulin secretion or both?

\section{Subjects and methods}

Subjects. Our study was part of a large base-line epidemiological survey of middle-aged men living in four municipalities of the Stockholm area. The overriding purpose of the survey is to serve as a basis for strategy and evaluation of prevention of Type II diabetes in the Stockholm Diabetes Prevention Programme [11].

Our study was designed as a population-based cross-sectional study. It was approved by the ethics committee of the Karolinska Hospital. It comprised a sample of men who were 35-54 years old in the year 1992. (Since data were assembled between 1992 and 1994, the actual age span of subjects at the time of data collection was 35 to 56 years). All subjects of the appropriate age who lived in the four municipalities Sigtuna,

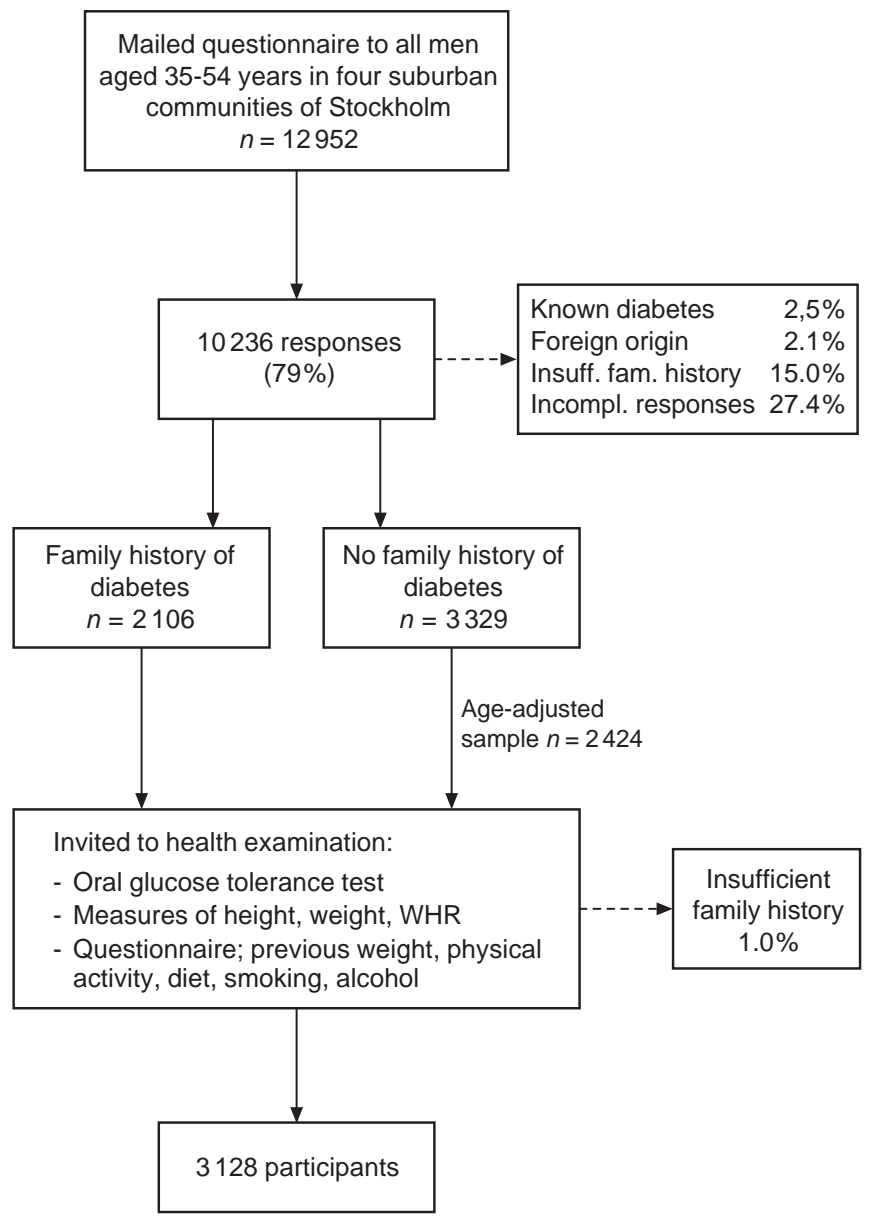

Fig. 1. Study design. For further details see subjects and methods

Tyresö, Värmdö and Upplands-Bro were included in the initial investigations. All four municipalities belong to the outer Stockholm area and are located between 20-40 kilometers from the city centre. The subjects to be studied were identified through the continuously updated population registry held by the Stockholm County Council which included all the inhabitants of the county.

An outline of the selection procedure is given in Figure 1. Sampling of subjects was by two sequential procedures. In the first, a short questionnaire was sent to all men in the appropriate age group living in the four municipalities. The questionnaire asked about country of birth and presence of diabetes in the subject and among relatives. Of the 12952 men who received the questionnaire answers were obtained from 10236 ( 79\%). The answers showed that 258 men $(2.5 \%)$ had diabetes known to themselves and that $212(2.1 \%)$ were born outside Sweden. These subjects were excluded from the rest of the study.

From remaining answers, we identified 2106 men with a family history of Type II diabetes specified as follows: known diabetes in at least two second-degree relatives (grandparent, uncle or aunt) or in at least one first-degree relative in the generation of the proband (sister or brother) or the preceding generation (fathers, mothers). Subjects reporting children with diabetes were not included in the family history group because of the likelihood that their family history indicated links to Type I (insulin-dependent) diabetes mellitus rather than to Type II diabetes. We furthermore identified 3328 subjects who did not have first or second-degree relatives (including children) nor 
Table 1. Characteristics of subjects with and without a family history of diabetes

\begin{tabular}{|c|c|c|c|c|c|c|c|c|}
\hline & \multicolumn{3}{|c|}{ Family history of diabetes } & \multicolumn{3}{|c|}{ No family history of diabetes } & \multirow[t]{2}{*}{ difference } & \multirow[t]{2}{*}{$p$-value } \\
\hline & $n$ & means & SD & $n$ & means & SD & & \\
\hline Height (m) & 1619 & 1.79 & 0.06 & 1504 & 1.80 & 0.06 & -0.01 & 0.003 \\
\hline Weight (kg) & 1621 & 85.1 & 12.9 & 1506 & 83.2 & 12.0 & 1.90 & $<0.001$ \\
\hline $\mathrm{BMI}\left(\mathrm{kg} / \mathrm{m}^{2}\right)$ & 1618 & 26.5 & 3.7 & 1503 & 25.7 & 3.3 & 0.78 & $<0.001$ \\
\hline WHR & 1616 & 0.91 & 0.01 & 1504 & 0.90 & 0.05 & 0.01 & $<0.001$ \\
\hline BP Syst. (mm/Hg) & 1610 & 125.7 & 14.7 & 1501 & 124.4 & 14.8 & 1.25 & 0.018 \\
\hline BP Dias. (mm/Hg) & 1610 & 80.4 & 9.6 & 1500 & 79.7 & 9.6 & 0.77 & 0.026 \\
\hline Age (years) & 1622 & 46.6 & 4.9 & 1507 & 46.7 & 4.9 & -0.03 & 0.841 \\
\hline
\end{tabular}

cousins with known diabetes. These subjects constituted the group without family history. We excluded from further investigations $1531(15.0 \%)$ men who did not fit either the family history or the non-family history category and 2800 men $(27.4 \%)$ who did not render complete answers on family history.

In the second procedure, all 2106 men who had given complete and positive information on their family history were contacted by telephone and asked to participate in further investigations. Along with the subjects with a family history we contacted in the same manner 2424 subjects of those without family history. The latter subjects were selected randomly within the 5 year age group of each participating subject with a family history. In total 3181 men $(70.2 \%)$ of those contacted agreed to participate.

The investigations took place at four local health care centres, one for each of the participating municipalities. The subjects to be studied reported to the local health care centre of their community in the morning (between 0700 and 0830 hours) after an overnight fast (starting at 2200 hours). Subjects were instructed to abstain from vigorous exercise in the evening before and in the morning of the investigations. Smokers were encouraged to abstain from smoking in the morning of the investigations. On arrival in the health care centre the information given by the subjects in the questionnaire on family history was first verified. As a result of this verification 52 subjects were found not to fit the family history or non-family history category and were therefore excluded from further studies. Altogether 3128 subjects constituted the final study group. In these subjects weight, height, and waisthip ratios were measured when the subjects were wearing light indoor clothing and no shoes. Blood pressure was measured in the supine position by a nurse.

Subsequent to these measures all of the 3128 subjects underwent a 75-g oral glucose tolerance test (OGTT) according to the World Health Organisation (WHO) criteria [12]. Venous blood samples were secured before and 120 min after the ingestion of glucose. Plasma samples were obtained after centrifugation and stored at minus $20^{\circ} \mathrm{C}$ until assays of glucose and insulin. During the OGTT the subjects were seated comfortably in calm surroundings. A detailed questionnaire which included eating habits, physical activity and social conditions was filled out at that time.

Assays. Concentrations of plasma glucose were assayed in duplicate using a glucose oxidase method and a Yellow Spring Glucose Analyser (Yellow Springs Inc., Yellow Spring, Ohio). Immunoreactive insulin (IRI) was assayed by RIA, using our own antibodies, human insulin as a standard and charcoal addition to separate antibody-bound and free insulin [13]. Proinsulin cross-reacts in this assay by about $80 \%$.

Calculations. Impaired glucose tolerance (IGT) and diabetes were defined according to WHO criteria, i.e. IGT was defined as a 2-h plasma glucose concentration between 7.8 and $11.0 \mathrm{mmol} / \mathrm{l}$ and diabetes above $11.0 \mathrm{mmol} / \mathrm{l}$. In the evaluation of insulin sensitivity and secretion a homeostasis model assessment (HOMA) was included. The HOMA model is based on ratios between fasting insulin and glucose values. The model is verified against near-steady-state C-peptide or insulin responses to hyperglycaemic clamps in non-diabetic people [9]. The HOMA variables of insulin secretion and sensitivity were calculated using the previously described algorithms [14] but excluding those constants which are dependent on the particular insulin assay used. Beta-cell function by HOMA was thus calculated as insulin $(\mathrm{mU} / \mathrm{l}) /[$ glucose $(\mathrm{mmol} / \mathrm{l})-3.5]$ and resistance as insulin $\times$ glucose.

Multiple logistic regression analysis was used to obtain the odds ratios (OR), accompanied by $95 \%$ confidence intervals (CI).Chi squared analysis was used to test for differences in distribution between insulin secretion and insulin resistance variables. All OR estimates were adjusted for age in two categories (35-45 and 46-56 years) and for BMI in three categories $\left(\leq 24.9,25.0-27.9\right.$ and $\left.\geq 28.0 \mathrm{~kg} / \mathrm{m}^{2}\right)$.

\section{Results}

General characteristics of subjects with or without a family history of diabetes. General characteristics are given in Table 1. Age was closely matched for the two groups studied. Height was slightly but statistically significantly lower in subjects with a family history. Subjects with a family history were, on average, slightly but significantly more obese than those without, whether assessed by body weight, BMI or by waist/ hip ratio (WHR). Diastolic blood pressure was slightly but statistically significantly higher in subjects with than in those without family history.

Subjects who reported a diabetic mother were 1.7 years older than those with a diabetic father (Table 2). Both systolic and diastolic blood pressure was significantly higher in subjects with a diabetic mother; however this could be explained, at least in part, by the age-related increase which amounted to $5.3 \mathrm{mmHg}$ for systolic and $2.0 \mathrm{mmHg}$ for diastolic blood pressure during a 10 year period. This age-related increase was similar in those with a diabetic mother and a diabetic father (data not shown). In other respects there were no significant differences between the two types of family history (Table 2). 
Table 2. Characteristics of subjects with and without a family history of diabetes

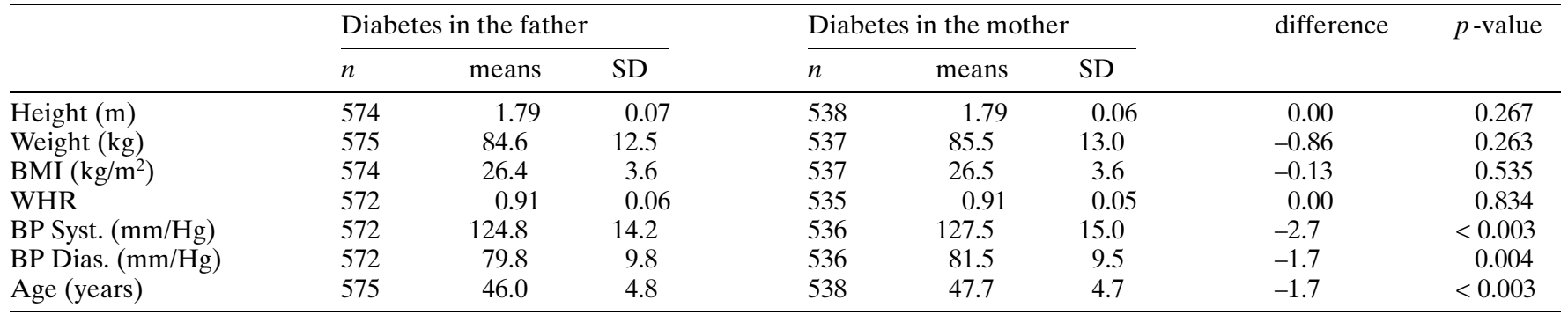

Table 3. Prevalence odds ratio (POR) of IGT and Type II diabetes associated with a family history of diabetes by number and closeness of relatives

\begin{tabular}{|c|c|c|c|c|c|c|c|}
\hline \multirow{2}{*}{$\begin{array}{l}\text { Family history } \\
\text { of diabetes }\end{array}$} & \multirow{2}{*}{$\begin{array}{l}\text { Normal GT } \\
n\end{array}$} & \multicolumn{3}{|c|}{ IGT } & \multicolumn{3}{|c|}{ Type II diabetes } \\
\hline & & $\bar{n}$ & POR & $95 \% \mathrm{CI}$ & $\bar{n}$ & POR & $95 \% \mathrm{CI}$ \\
\hline$\overline{\mathrm{No}}$ & 1433 & 60 & 1.0 & & 10 & 1.0 & \\
\hline Yes & 1460 & 112 & 1.6 & $1.2-2.3$ & 45 & 4.1 & $2.1-8.3$ \\
\hline No & 1433 & 60 & 1.0 & & 10 & 1.0 & \\
\hline Two second-degree relatives & 201 & 11 & 1.1 & $0.6-2.2$ & 3 & 2.1 & $0.6-7.6$ \\
\hline More than two second-degree relatives & 76 & 4 & 1.4 & $0.5-3.9$ & 2 & 4.3 & $0.9-20.2$ \\
\hline One first-degree relative & 1042 & 79 & 1.7 & $1.2-2.3$ & 34 & 4.4 & $2.1-8.9$ \\
\hline More than one first-degree relative & 140 & 18 & 2.4 & $1.3-4.2$ & 6 & 5.0 & $1.8-14.1$ \\
\hline
\end{tabular}

Table 4. Prevalence odds ratio (POR) of IGT and Type II diabetes associated with a family history of diabetes by gender of relatives

\begin{tabular}{|c|c|c|c|c|c|c|c|}
\hline \multirow{2}{*}{$\begin{array}{l}\text { Family history } \\
\text { of diabetes }\end{array}$} & \multirow{2}{*}{$\begin{array}{l}\text { Normal GT } \\
n\end{array}$} & \multicolumn{3}{|c|}{ IGT } & \multicolumn{3}{|c|}{ Type II diabetes } \\
\hline & & $n$ & POR & $95 \% \mathrm{CI}$ & $n$ & POR & $95 \% \mathrm{CI}$ \\
\hline No & 1433 & 60 & 1.0 & & 10 & 1.0 & \\
\hline Father's side & 520 & 39 & 1.7 & $1.1-2.5$ & 15 & 4.1 & $1.8-9.3$ \\
\hline Mother's side & 560 & 41 & 1.6 & $1.0-2.4$ & 18 & 4.1 & $1.9-9.1$ \\
\hline Both father's and mother's side & 301 & 28 & 1.9 & $1.2-3.1$ & 10 & 4.6 & $1.9-11.1$ \\
\hline No & 1433 & 60 & 1.0 & & 10 & 1.0 & \\
\hline Father & 514 & 44 & 1.9 & $1.3-2.9$ & 16 & 4.4 & $2.0-9.9$ \\
\hline Mother & 486 & 33 & 1.5 & $0.9-2.3$ & 17 & 4.4 & $2.0-9.8$ \\
\hline Both father and mother & 63 & 11 & 3.1 & $1.5-6.3$ & 5 & 8.5 & $2.8-26.2$ \\
\hline No & 1433 & 60 & 1.0 & & 10 & 1.0 & \\
\hline Female relatives & 464 & 28 & 1.3 & $0.8-2.0$ & 13 & 3.5 & $1.5-8.1$ \\
\hline Male relatives & 454 & 34 & 1.7 & $1.1-2.6$ & 17 & 5.2 & $2.4-11.5$ \\
\hline Both female and male & 541 & 50 & 1.9 & $1.3-2.9$ & 15 & 3.8 & $1.7-8.6$ \\
\hline
\end{tabular}

Prevalence of diabetes and IGT in relation to number and closeness of relatives with diabetes. Diabetes was diagnosed in 55 (1.8\% of the study population ) and impaired glucose tolerance (IGT) in 172 subjects $(5.5 \%)$. When adjusted for age and BMI the OR associated with family history was 4.1 or diabetes and 1.6 for IGT (Table 3). The relative risk of diabetes and IGT increased with the number and closeness of relatives with diabetes. Thus, those with more than one first-degree relative with diabetes tended to have the highest OR.

Table 4 compares the prevalence of IGT and diabetes in relation to gender-specific relatives. The ORs associated with female relatives did not differ from those of male relatives.
Association between a family history of diabetes and fasting plasma glucose. Within the normal range of fasting plasma glucose (i. e. $<7.8 \mathrm{mmol} / \mathrm{l}$ ) there was an association between family history and plasma glucose concentrations in the upper half of normal values (Table 5). An association with family history was already noted in the interval of fasting glucose levels between 4.7 and $5.5 \mathrm{mmol} / 1$ relative to lower glucose concentrations.

Association of a family history of diabetes with 2-h glucose values in subjects with normal glucose tolerance (NGT). The mean 2-h glucose values of subjects with normal 2-h glucose values (NGT) were higher in subjects with than without a family history. The OR 
Table 5. Effects of a family history of diabetes on fasting plasma glucose concentrations

\begin{tabular}{|c|c|c|c|c|c|c|c|c|c|c|}
\hline \multirow{3}{*}{$\begin{array}{l}\text { Family history } \\
\text { of diabetes }\end{array}$} & \multicolumn{10}{|c|}{ Glucose concentration $(\mathrm{mmol} / \mathrm{l})$} \\
\hline & \multirow{2}{*}{$\begin{array}{l}\leq 4.6 \\
n\end{array}$} & \multicolumn{3}{|c|}{$4.7-5.5$} & \multicolumn{3}{|c|}{$5.5-6.7$} & \multicolumn{3}{|c|}{$\geq 6.8$} \\
\hline & & $n$ & POR & $95 \% \mathrm{CI}$ & $n$ & POR & $95 \% \mathrm{CI}$ & $n$ & POR & $95 \% \mathrm{CI}$ \\
\hline No & 771 & 594 & 1.0 & & 125 & 1.0 & & 13 & 1.0 & \\
\hline Yes & 701 & 672 & 1.2 & $1.0-1.4$ & 208 & 1.7 & $1.3-2.1$ & 37 & 2.8 & $1.5-5.3$ \\
\hline
\end{tabular}

of having 2-h glucose values of 5.8-7.7 mmol/1 (compared with glucose levels $<3.8 \mathrm{mmol} / \mathrm{l}$ ) was thus increased to 1.4 (CI 1.1-1.7), that of $4.8-5.7$ to 1.3 (CI $1.0-1.6)$ and that of 3.8-4.7 not significantly to 1.1 (CI 0.9-1.3).

The OR of having 2-h glucose values in the range of 5.8-7-7 mmol/l (compared with glucose concentrations $<3.8 \mathrm{mmol} / \mathrm{l}$ ) were compared in subjects with a mother compared with those with a father as a diabetic relative and found not to differ significantly (OR 1.5, CI 1.1-1.9 for mother and 1.1, CI 0.9-1.5 for father as diabetic relative).

Family history of diabetes in combination with obesity in relation to IGT and diabetes. Obesity is the most thoroughly documented risk factor for diabetes and IGT [15]; it was therefore adjusted for in the data analysed above. We also wished to assess the interrelationship between overweight and a family history for relative risks of diabetes and IGT. Subjects were divided into those with BMI less than $25 \mathrm{~kg} / \mathrm{m}^{2}$ and those above. Subjects with a family history and high BMI had a mean 24-fold increased OR of diabetes compared with leanness and no family history (Fig.2). The association between obesity and IGT was as strong as for overt diabetes, whereas the influence of a family history appeared less pronounced (Fig. 2).

Association of family history of diabetes with beta-cell function and insulin sensitivity. Because glucose tolerance per se is known to affect insulin release and sensitivity, we assessed the variables of insulin secretion and sensitivity in different strata of glucose tolerance as determined from the results of OGTT. Four different concentrations of glucose tolerance were defined: 2-h plasma glucose concentrations below $5.8 \mathrm{mmol} / \mathrm{l}$, those between 5.8 and $7.7 \mathrm{mmol} / \mathrm{l}$, IGT and diabetes.

The difference in proportion of a family history in relation to no family history for the chosen variables of insulin sensitivity and secretion are shown in Table 6. Among subjects with normal glucose tolerance, those with a family history displayed lesser sensitivity to glucose, as judged from HOMA and fasting insulin concentrations. This was in marked contrast to the subjects in whom diabetes was discovered during the investigation. In the diabetic subjects the frequency of insulin resistance was thus lower in subjects with a family history than in those without (Table 6). Recip-

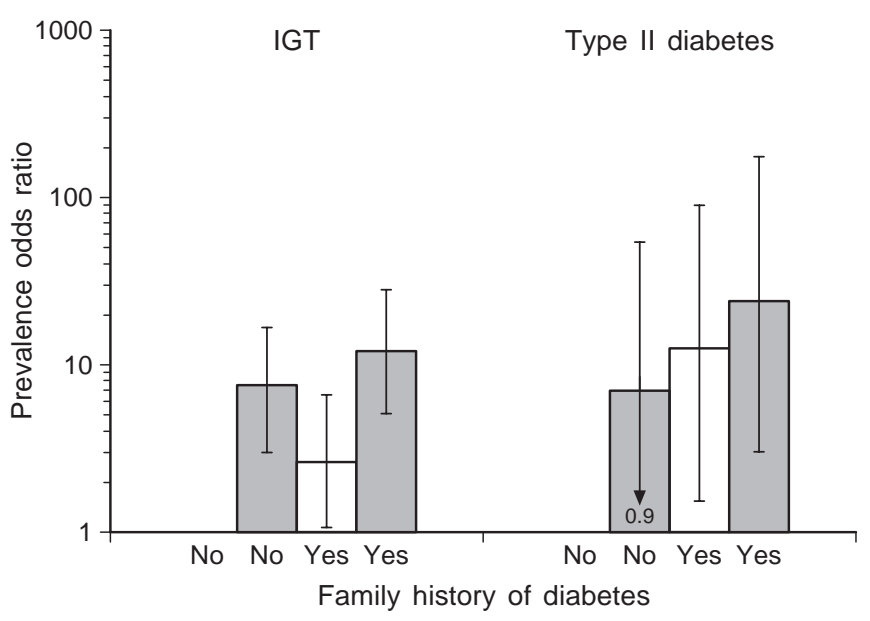

Fig. 2. Odds ratios of IGT and diabetes associated with combinations of BMI and family history of diabetes. The $95 \%$ confidence intervals are indicated by bars. $\square$ BMI $\leq 24,9$; $\mathrm{BMI} \geq 25,0$

rocally, low insulin secretion tended to be more frequent in diabetic subjects with a family history compared with diabetic subjects without (Table 6).

The corresponding analysis comparing insulin sensitivity and secretion in subjects with a diabetic mother compared with subjects with a diabetic father did not yield any significant differences (results not shown).

\section{Discussion}

To our knowledge, this is the largest study in a lowprevalence population which investigates the impact of a family history of diabetes on glucose tolerance and Type II diabetes. The study should be representative of ethnically Swedish men, since participation rate was high and subjects who were obviously not of natural Swedish origin were excluded. Our data indicate a strong and multifactorial influence of family history of diabetes at all levels on glucose tolerance in this population, without, however, evidence for a gender effect.

Several methodological questions must be answered before the results can be interpreted properly. One question pertains to the reliability of the recall procedure. Recall bias are likely to occur in subjects with known diabetes compared with non-diabetic 
Table 6. Distribution of subjects with a family history compared with no family history of diabetes in relation to variables of insulin resistance and insulin secretion

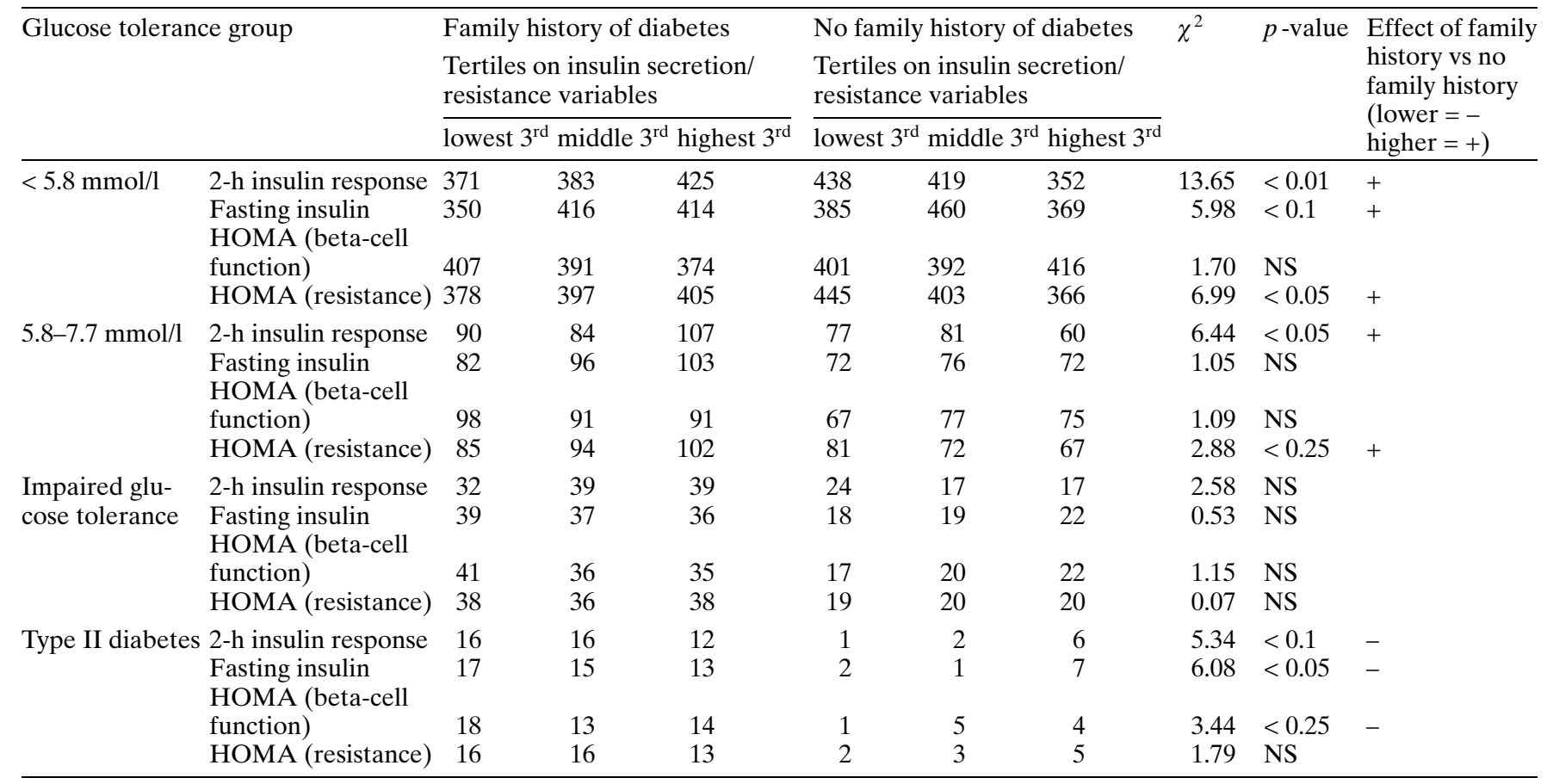

subjects, since the former are likely to recall family history better than the latter. It was therefore an important advantage of the present study design that participating subjects were, by definition, unaware of diabetes in themselves before the investigations. Furthermore, we made considerable efforts to minimize incorrect recall. Hence, participating subjects were asked about family history on two separate occasions, first by questionnaire, then, several weeks later, by interview at the time of OGTT. More importantly, we excluded a considerable number of subjects ( $27 \%$ of the primary study group) who were unable to fully account for presence or absence of diabetes among relatives. It is of course realized that these measures do not completely guarantee a correct recall. Furthermore, the error of undiagnosed diabetes in relatives of the study subjects has to be considered. In a recent population study in Sweden the presence of undiagnosed diabetes was thus about $50 \%$ of the frequency of known diabetes [16].

A further question arises as to the type of diabetes the subjects with diagnosed disease had. The age span investigated (35-54 years) makes it likely that most subjects in whom we diagnosed hyperglycaemia had Type II diabetes. Also the clinical follow-up of the subjects diagnosed with diabetes made this likely: all had mild diabetes and none needed insulin treatment during observation time for at least a year (results not shown).

A related question is whether a family history of diabetes pertains to Type I or Type II diabetes. To minimize inclusion of Type I diabetes in family history, we purposely excluded those of our subjects who reported children with diabetes, since family history in those instances was likely to be linked to Type I rather than to Type II diabetes. In separate analysis of the investigated subjects with a family history, we restricted the analysis to those who reported that relatives of their own or of older generations were diabetic after the age of 35. This analysis, however, yielded essentially the same results as those presented above, i.e. the relation between a family history and glucose tolerance and variables of insulin secretion or insulin resistance were not altered (results not shown).

It is important to note that we analysed family history in relation to glucose tolerance only in subjects not previously known to have diabetes. This design has advantages as well as limitations. An advantage is that relations between family history and external risk factors, such as overweight, are not influenced by therapeutic efforts and knowledge about the disease. A limitation is that the impact of family history that we observed in overt diabetes is restricted to the incidences discovered during the OGTT. One consequence of this could be that what we study here is the relation of a family history to mild cases of Type II diabetes since more severe diabetes would produce symptoms prompting investigations and earlier diagnosis. The design possibly selected against adult Type I diabetes, since this form of the disease usually presents with severe diabetes.

A fundamental problem is to ascertain whether a family history relates to heredity or to family-shared conditions, such as social class, family values, educa- 
tional levels, eating habits etc. In our study, we have accounted for the influence of overweight (discussed below) which is a major risk factor that could, in part, be related to conditions during childhood and adolescence. That other factors encompassed by "social heredity" are important for the impact of a family history on IGT and diabetes is suggested by a previous study [17]. These other factors are to be explored in future analysis of our population. Another factor of potential importance is fetal environment, since it has been documented that low birth weight associates with diabetes [18]. Low height is associated with low birthweight and it is therefore interesting that in our study the height of subjects with family history was slightly, but significantly lower than in those without a family history. The possible influence of birthweight in our study population is currently under investigation.

We find that family history was inversely associated with glucose tolerance over the entire range of 2-h glucose values during the OGTT. The association with a family history was thus strongest for diabetes, followed by IGT. Within the NGT range of glucose concentrations associations with family history diminished successively from higher to lower glucose values. This finding is consistent with, but does not prove, that the "dosage" of genetic factors determines the level of glucose tolerance in a given person.

The proportion of diabetic subjects with a family history displaying low indices of insulin secretion tended to be increased relative to diabetic subjects without. Reciprocally, the proportion of diabetic subjects with a family history displaying insulin resistance was decreased relative to diabetic subjects without. These findings agree with data from a group of much older Type II diabetes subjects in the Netherlands [19]. These results suggest that the diabetogenicity of family history is linked to negative effects on insulin secretion. It is clear, however, that our data do not rule out potential influences of a family history on insulin sensitivity. In fact, subjects with a family history and normal glucose tolerance displayed signs of insulin resistance. Only a prospective follow-up study can determine the relative importance of insulin resistance and low insulin secretion for development of diabetes in our study group.

The strength of the present assessments of insulin sensitivity and secretion could be questioned on the grounds that the variables measured are crude but, although simple, they appear basically valid. At normoglycaemia raised fasting concentrations of insulin (or immunoreactive insulin) are a widely used measure of insulin resistance. Also, the HOMA analysis has been used in large prospective studies, such as the UK prospective one in Type II diabetic subjects [20]. The HOMA analysis is based on the relation between fasting insulin and glucose values [14]. To some extent at least, HOMA has been validated as to insu- lin sensitivity and insulin deficiency [21]. The 2-h insulin values are more difficult to interpret, since high concentrations can occur either as a result of insulin resistance or as a result of delayed stimulation of insulin secretion by glucose [22]. However, the association of a family history of diabetes with the lowest tertile of 2-h insulin concentrations at raised 2-h glucose concentrations can only be interpreted as an association with low insulin secretion.

Type II diabetic patients have raised proinsulin to insulin ratios in circulation [23] and this effect has been proposed to be genetically determined. That our RIA co-measures proinsulin with insulin could affect, to some extent, the insulin measurements in the Type II diabetic subjects but this does not explain the relation observed between insulin secretion in diabetic subjects with or without a family history of diabetes. The co-measurement of proinsulin is not likely to add to insulin measurements to a major extent in remaining subjects, since the percentage of fasting proinsulin in relation to insulin is low (below 15\%) $[23,24]$.

Diabetes in the mother as compared with diabetes in the father was found previously to increase Type II diabetes in the offspring of animals [25] and man $[6-9,26]$. This association has been proposed to be due to influences of maternal diabetes on the foetus. A preferential association of non-insulin-dependent diabetes in the offspring with maternal compared with paternal inheritance was also seen, however, when diabetes in the mother was apparent only after the pregnancy. Mutations in mitochondrial genes [27] could give rise to such an association as could, at least theoretically, genetic imprinting. The notion of a preferential impact of the mother's diabetes occurring after pregnancy is, however, controversial as one study failed to document an association between maternal diabetes and diabetes in male Caucasian offspring [10].

In our population we did not observe any association between a family history of diabetes and gender, nor specifically between family history in mother compared with father. Neither did we detect an influence of maternal diabetes on obesity-related variables in our subjects. The reasons for discrepancies between different studies are not known. It seems possible that a putative maternal influence could give rise to diabetes of early onset and of such severity as to be detected before the 35-54 year age of our population. It should also be noted that the maternal association was, in one study, strongest for patients who were considerably older than our subjects [7].

Obesity is the strongest non-hereditary risk factor for Type II diabetes $[1,15]$. Our results show added effects between a family history and obesity for risk of diabetes. Such effects have also been observed previously [28] but not, as in our study, in a subject sample drawn from the general population. Interestingly, 
the obesity- associated relative risk for IGT was similar to the OR for diabetes, whereas the OR conferred by a family history was stronger for diabetes than for IGT. These observations could indicate that moderate obesity per se is sufficient to cause IGT but that additional, hereditary, factors are needed to cause diabetes.

The association between a family history and diabetes was very strong in our study. One reason for this could be the exclusion of even a minor family history (such as third degree relatives) from the control (no family history) group as well as all subjects who were unable to give complete information on family history. In this way we have minimized (but not excluded) the presence of undiagnosed family history in the non-family history group. Another reason for the strong association could be due to the relatively young age at which diabetes was discovered in our subjects. The aging process is known to involve both a decrease in insulin sensitivity and in insulin secretion [29]. If the sum of negative hereditary and non-hereditary factors determine onset of diabetes, then more hereditary factors are needed for diabetes debut at a younger than at an older age. Experimental studies support this notion [30]. On follow-up of this cohort we would then expect new cases of diabetes to display a weaker association with a family history than the subjects in whom diabetes was presently discovered.

Finally, our results imply that middle-aged men with both a family history and obesity should be screened in the general population. This is because of the very high risk of unrecognized diabetes that we found in such subjects and the possibility of therapeutic intervention. As shown here, such screening can easily be done by a short postal questionnaire.

In summary, the present study has shown a strong effect of a family history of diabetes on glucose tolerance. The effect is graded as to number and closeness of relatives. The impact of a family history on glucose tolerance is a continuous variable, being strongest for diabetes, less strong for IGT but detectable also in a higher strata within the normal range of 2-h postglucose values. No differences were seen between a family history on maternal and paternal side as to OR for decreased glucose tolerance or diabetes-related variables. The data also indicate that diabetogenic heredity in a Swedish population includes as a major component a decreased capacity for insulin secretion. Lastly, the synergy shown between heredity and obesity as risk factors emphasize the need for interventive efforts to prevent diabetes in subjects displaying both obesity and a family history of diabetes.

Acknowledgements. The study was financed by a grant from the Stockholm County Council. We thank the many nurses and other staff members who carried out this investigation in practice. Special thanks are due to the coordinating efforts of Ms C. Ekehjelm.

\section{References}

1. Hamman R (1992) Genetic and environmental determinants of non-insulin-dependent diabetes mellitus (NIDDM). Diabetes/Metabolism Reviews 8: 287-338

2. Saad M, Knowler W, Pettit D, Nelson R, Mott D, Bennett P (1984) The natural history of impaired glucose tolerance in the Pima Indians. N Engl J Med 319: 1500-1506

3. King H, Zimmet P, Raper L, Balkau B (1984) Risk factors for diabetes in three Pacific populations. Am J Epidemiol 119: 396-409

4. Haffner S, Stern M, Hazuda H, Pugh H, Patterson J (1986) Hyperinsulinemia in a population at high risk for non-insulin dependent diabetes mellitus. N Engl J Med 315: 220-224

5. Sartor G (1984) Prevalence of type 2 diabetes in Sweden. Acta Endocrinol Suppl 262: 27-29

6. Alcolado J, Alcolado R (1991) Importance of maternal history of non-insulin-depdendent diabetic patients. BMJ 302: $1178-1180$

7. Thomas F, Balkau B, Vauzelle-Kervoedan F, Papoz L (1994) Maternal effect and familial aggregation in NIDDM. The CODIAB study. Diabetes 43: 63-67

8. Simmons D, Gatland B, Leakehe L, Fleming C (1995) Frequency of diabetes in family members of probands with non-insulin-dependent diabetes mellitus. J Int Med 237: 315-321

9. Riley M, Blizzard C, McCarthy D, Senator G, Dwyer T, Zimmet P (1997) Parental history of diabetes in an insulin-treated diabetes registry. Diabetic Med 14: 35-41

10. Mitchell B, Valdez R, Hazuda H, Haffner S, Monterosa A, Stern M (1993) Differences in the prevalence of diabetes and impaired glucose tolerance according to maternal or paternal history of diabetes. Diabetes Care 16: 1262-1267

11. Bjärås G, Ahlbom A, Alvarsson M et al. (1998) Strategies and methods for implementing a community-based diabetes primary prevention program in Sweden. Health Promotion 12: 151-160

12. World Health Organization Study Group on Diabetes Mellitus (1985) Technical report series, No 727, WHO, Geneva, pp 9-13

13. Herbert V, Lau KS, Gottlieb CW, Bleicher SJ (1965) Coated charcoal immunoassay of insulin. J Clin Endocrinol Metab 25: 1375-1384

14. Matthews D, Hosker J, Naylor B, Treaches D, Turner R (1985) Homeostasis model assessement insulin resistance and B-cell function from fasting plasma glucose and insulin concentrations in men. Diabetologia 28: 412-419

15. Pedersen O (1989) Impact of obesity on the pathogenesis of non-insulin-dependent diabetes mellitus: a review of current hypothesis. Diabetes Metab Rev 5: 495-509

16. Andersson D, Svärdsudd K, Tibblin G (1991) Prevalence and incidence of diabetes in a Swedish community 1972-1987. Diabetic Medicine 8: 428-434

17. Leonetti D, Fujimoto W, Wahl P (1989) Early-life background and the development of non-insulin-dependent diabetes mellitus Am J Phys Anthropol 9: 345-355

18. Hales C, Barker D (1992) Type 2 (non-insulin-dependent) diabetes mellitus, the thrifty phenotype hypothesis. Diabetologia 35: 595-601

19. Boer J, Feskens E, Kromhout D (1996) Characteristics of non-insulin-dependent diabetes mellitus in elderly men: effect modification by family history. International Journal of Epidemiology 25: 394-402

20. UK Prospective Diabetes Study Group (1995) UK prospective diabetes study 16 . Overview of 6 years' therapy of type II diabetes: a progressive disease. Diabetes 44: 1249-1258 
21. Haffner S, Gonzalez C, Miettinen H, Kennedy E, Stern M (1996) A prospective analysis of the HOMA model. The Mexico City diabetes study. Diabetes Care 19: 1138-1141

22. Pimenta W, Mitrakou A, Jensen T, Yki-Järvinen H, Daily G, Gerich J (1996) Insulin secretion and insulin sensitivity in people with impaired glucose tolerance. Diabetic Medicine 13 [Suppl 6]:S33-S36

23. Davis S, Alberti K (1991) Proinsulin. In: Alberti K, Krall L (eds) The Diabetes Annual/6, Elsevier Science Publishers B. V., pp 409-430

24. Grill V, Pigon J, Hartling S, Binder C, Efendic S (1990) Effects of dexamethasone on glucose-induced insulin and proinsulin release in low and high insulin responders. Metabolism 39: 251-258

25. Aerts L, Holemans K, Van Assche F (1990) Maternal diabetes during pregnancy: consequence for the offspring. Diabetes/Metab Rev 6: 147-76
26. Petitt D, Aleck K, Baird H, Carraher M, Bennett P, Knowler W (1988) Congenital susceptibility to NIDDM: role of intrauterine environement. Diabetes 37: 622-628

27. Maasen J, Kadowaki T (1996) Maternally inherited diabetes and deafness: a new diabetes subtype. Diabetologia 39: 375-383

28. Morris R, Rimm D, Hartz A, Kalkhoff R, Rimm A (1989) Obesity and heredity in the etiology of non-insulin-dependent diabetes mellitus in 32.662 adult white women. American J Epidemiol 130: 112-121

29. Chen M, Bergman R, Pacini G et al. (1985) Pathogenesis of age-related glucose intolerance in man: insulin resistance and decreased B-cell function. J Clin Endocrinol Metab 60: $13-20$

30. Mitchell B, Kammerer C, Reinhart L, Stern M (1994) NID$\mathrm{DM}$ in Mexican-American families. Heterogeneity by age of onset. Diabetes Care 17: 567-573 